\title{
Pegylated Curcumin with Gold Nanoparticles: Antimicrobial Agent Evaluation
}

\author{
Daniella dos Santos Courrol'1, Bruna Henrique Teixeira², Camila Bueno Pacheco Pereira1, Marcia \\ Regina Franzolin'1, Lilia Coronato Courrol ${ }^{2}$ \\ 1Instituto Butantan, Laboratório de Bacteriologia, São Paulo, SP, Brazil. \\ 2Universidade Federal de São Paulo, Laboratório de Lasers e Óptica Biomédica Aplicada, Departamento de Ciências \\ Exatas e da Terra, Diadema, SP - Brazil \\ lccourrol@gmail.com
}

\begin{abstract}
Curcumin is a polyphenol derived from the Curcuma longa plant. Curcumin exhibits a variety of therapeutic properties, including antioxidant, analgesic, anti-inflammatory, anticancer and antiseptic activities. Curcumin acts as photosensitizer in photodynamic therapy (PDT), which is an alternative way to kill pathogenic microorganisms. The use of gold nanoparticles (AuNPs) as the vehicle for curcumin delivery increases photodynamic ability. Therefore, the aim the present study is to investigate the effect of the photodynamic action of LED-activated curcumin gold nanoparticles, on cell viability of Staphylococcus aureus (Sa), Staphylococcus epidermidis (Se), Pseudomonas aeruginosa (Pa), Escherichia coli (Ec), Citrobacter freundii $(\mathrm{Cf})$ and Klebsiella pneumoniae (Kp). To prepare Curcumin gold nanoparticles (Curc:AuNps) solutions, $3.2 \mathrm{mg}$ of $\mathrm{HAuCl}_{4}$ was mixed with $1.5 \mathrm{mg}$ Curcumin and Polyethylene glycol (PEG) in Mili-Q water. This solution was exposed to light from a mercury metal halide lamp. The resulting solutions were characterized by UV-Vis and transmission electron microscopy (TEM). The in vitro antibacterial activity of Curc:AuNps was evaluated against Sa, $\mathrm{Se}, \mathrm{Pa}, \mathrm{Ec}$, Cf and Kp using the broth dilution assay. The effects of exposure to yellow LED light with $\sim 38 \mathrm{~J} / \mathrm{cm}^{2}$ fluence and variations in the Curc:AuNPs concentrations (38, 50, 75 and $100 \%$ dilutions) were studied. The presence of a surface plasmon resonance band at $\sim 560 \mathrm{~nm}$ indicated the formation of spherical gold nanoparticles. In the presence of PEG, the solution color changed some minutes after the mixing of reagents, and solution size homogeneity was improved with the mercury metal halide lamp illumination. TEM analyses showed $\sim 17 \pm 2 \mathrm{~nm}$ nanoparticles. The results indicate that PDT with curcumin gold nanoparticles inhibits the development of Gram positive and Gram negative bacteria. This study revealed antibacterial probability of inhibition $>80 \%$ with $32 \mathrm{mM}$ of Curc:AuNPs and light against $\mathrm{Sa}, \mathrm{Se}, \mathrm{Cf}$ and $\mathrm{Kp}$ strains after 18 hours of incubation. NOVA/Tukey's tests were conducted to compare groups curcumin/Curc:AuNPs, with and without photodynamic excitation.
\end{abstract}

Keywords: Gold nanoparticles, curcumin, photodynamic therapy, antibacterial.

(C) Copyright 2016 Authors - This is an Open Access article published under the Creative Commons Attribution License terms (http://creativecommons.org/licenses/by/3.0). Unrestricted use, distribution, and reproduction in any medium are permitted, provided the original work is properly cited.

\section{Introduction}

Curcumin (diferuloylmethane), is a polyphenol that accumulates in the rhizome of Curcuma longa, can reduce the risk of heart disease, high blood pressure and cognitive decline ${ }^{1-8}$. Additionally, due to its capabilities to regulated important transcription factors, protein kinases, cytokines, adhesion molecules and redox status, curcumin can serve as an anti-inflammatory, antioxidant, anti-angiogenic, antimicrobial and antineoplasic agent ${ }^{9}$.

Despite progress in development of antibacterial agents, there are still special needs to find new antibacterial agents due to development of multidrug resistant bacteria. Curcumin has been shown to inhibit cellular proteins $\mathrm{FtsZ}^{10}$ and sortase $\mathrm{A}^{11}$, thereby interrupting cytokinesis and cellular adhesion, and interfere with biofilm formation ${ }^{12}$. Though curcumin exhibits broad antimicrobial activity, some data suggest that Gram-positive species are more susceptible than Gram-negatives ${ }^{1}{ }^{9}$. In adition, Antibiotics are sometimes associated with adverse effects on the host including hypersensitivity, immune-suppression and allergic reactions. Curcumin described as prebiotic, which have beneficial effects on human health. 
In this paper the antibacterial properties of curcumin will be evaluated and alternatives to increase these properties will be studied: the synthesis of gold nanoparticles using curcumin as reductor agent and uses of light to stimulate photodynamic properties of curcumin.

Studies shown that the bioavailability of curcumin can be increased with its conjugation on the surface of gold nanoparticles. Metal nanoparticles can be produced by chemical reduction methods, physical methods as photoreduction ${ }^{13}$, microwave dielectric heating ${ }^{14}$, ultrasonic irradiation ${ }^{15}$, and radiolysis ${ }^{16}$, and biological routes ${ }^{17}$. Electromagnetic radiation is a green alternative to the synthesis of nanoparticles, since it is free of environmentally negative effects, such as use of toxic solvents or aggressive reducers ${ }^{18}$. The use of light in the synthesis of nanoparticles have emerged in 1999 with Zhou et al. 19, when shape-controlled gold nanoparticles were synthesized using ultraviolet irradiation technique at room temperature. Dong et al. 20 used sunlight to synthesize nanoparticles of gold in suspension. Tomita et al. obtained silver nanoparticles with Tryptophan and light and observed its lethal effect against bacteria ${ }^{21}$. Mao et al. ${ }^{22}$ obtained size and shape deviations of $<6 \%$ of the gold nanoparticles under optimal ultraviolet irradiation and chemical conditions.

Gangwar et al. in 201223, reported the formation of curcumin conjugated with gold nanoparticles using Polyvinyl pyrrolidone which has been used as drug carrier for various drugs. In 2013 Dheeraj et al. synthesized the gold nanoparticles (AuNPs) by direct reduction of chloroauric acid $\left(\mathrm{HAuCl}_{4}\right)$ using curcumin in an aqueous phase without the use of any other reducing agents. Sreelakshm et al. 24 synthesized gold nanoparticles using turmeric rhizome extract, curcumin and curcumin glycoside acting as reducing and stabilizing agents at room temperature. They observed that the carbonyl group of enolic curcumin helps in reduction as well as stabilization of gold nanoparticles. Shlar et al. ${ }^{23,} 25$ prepared waterdispersible curcumin nanoparticles by bottom-up antisolvent precipitation approach and observed a minimal inhibitory concentration of 400 or 500 ? toward Escherichia coli.

Curcumin also acts as photosensitizer in Photodynamic therapy (PDT) [14], an alternative way to kill pathogenic microorganisms. In PDT, photosensitizers, are activated by visible light to produce cytotoxic reactive oxygen species (ROS), which induce lethal damages on target cells and tissues [5-8]. In comparison to conventional antibiotic treatment, PDT has unique advantages of dual specificity in targeting eradication of pathogenic microorganisms: preferential absorption of pathogenic microorganisms and target lesion irradiated from laser or visible light. Furthermore, the resistance of bacteria to PDT is very unlikely because of the nonspecific damage of PDT on bacteria [12]. So, PDT has shown potential promise in eradicating pathogenic microorganisms and treating infectious diseases. The use of AuNPs as the vehicle for curcumin delivery represents a promising approach, especially if the immobilization of curcumin on the particle surface be better for ROS formation. Therefore, in the present study our aim is also to investigate the effect of photodynamic action of LED-activated curcumin on cell viability of Staphylococcus aureus, Staphylococcus epidermidis, Pseudomonas aeruginosa, Escherichia coli, Citrobacter freundii and Klebsiella pneumoniae, in the presence of gold nanoparticles.

\section{Materials and Methods}

\subsection{Gold nanoparticles generation and evaluation}

Chloroauric acid, HAuCl4 99\%, CAS 27988-77-8) ( 1.5mg), Polyethylene Glycol (PEG) 10000 (10mg/m) and Curcumin (3.2 mg) from Curcuma Longa (CAS 45837-7) were purchased from Sigma-Aldrich. No other chemical reagents were used in the synthesis. The gold nanoparticles (CurcAuNPs) were produced by mixing the reagents in $100 \mathrm{~mL}$ of miliQ water solution, followed by vigorous stirring and illumination with a metal halide bulb lamp (Leica EL6000), not focused and placed $10 \mathrm{~cm}$ from the sample reservoir for $15 \mathrm{~min}$.

UV-vis absorption spectra were recorded by a Shimadzu Multispec-1501 UV-vis spectrophotometer using 10-mm quartz cells.

The morphology of the curcumin nanoparticles (Curc:AuNPs) was determined by transmission electron microscopy using a LEO 906E instrument from Zeiss, Germany $(6 \mu \mathrm{A}$ and $80 \mathrm{kV})$. For the measurements, a drop of gold nanoparticles $(5 \mu \mathrm{L})$ dispersed in bideionized water was placed onto a carbon-coated copper grid. The excess liquid was removed using a paper wick, and the deposit was dried in air for $5 \mathrm{~min}$ prior to imaging. The images were captured by a Megaview III camera and processed using the iTEM universal TEM imaging platform (Olympus Soft Imaging Solutions GmbH, Germany). 


\subsection{Broth Microdilution Assay for the determination of antimicrobial activity}

The microorganisms used in this assay were: Staphylococcus aureus American Type Culture Collection (ATCC) 25923, Staphylococcus epidermidis (clinical sample), Pseudomonas aeruginosa ATCC 27853, Escherichia coli ATCC 25922, and the clinical samples of Citrobacter freundii and Klebsiella pneumoniae.

The antimicrobial activity of the tested substances was determined by 2 -fold serial broth microdilution in culture medium in 96-well plates. The assays were conducted according to the guidelines of the National Committee for Clinical Laboratory Standards (NCCLS. Performance Standards for Antimicrobial Susceptibility Testing; Twenty-Fifty Informational Supplement. (NCCLS, 2012), for determination of Minimal Inhibitory Concentration (MIC). Aliquots of 50 $\mu \mathrm{l}$ of Mueller-Hinton broth (Difco) with bacterial inoculum adjusted to approximately $10^{6} \mathrm{CFU} / \mathrm{ml}$ was applied in the microplates containing $50 \mu \mathrm{l}$ of the diluted solutions, resulting in a final volume of $100 \mu \mathrm{l}$ with $10^{4}$ $\mathrm{CFU} /$ well. A positive growth control containing only culture medium and the strains under study was also prepared. The $100 \mu \mathrm{L} \mathrm{MH}$ liquid culture medium was used as negative control in the study. The nanoparticles concentrations used were: $38 \% \quad(11.3 \mu \mathrm{g} / \mathrm{mL})$, $50 \%(15.0 \mu \mathrm{g} / \mathrm{mL}), \quad 75 \% \quad(22.5 \mu \mathrm{g} / \mathrm{mL})$ and $100 \%$ $(30.0 \mu \mathrm{g} / \mathrm{mL})$.

After inoculation, the plates were incubated at $37^{\circ} \mathrm{C}$ for $18 \mathrm{~h}$ before the results were recorded. After this time, the turbidity of the cultures was measured in an ELISA reader (Multiskan ${ }^{\circledR} \mathrm{EX}$ (Thermo Isher Scientific, EUA) at $595 \mathrm{~nm}$ to assess bacterial growth. The results were expressed as inhibition percentage of optical density (OD) against a control; this control was obtained in each situation by measuring the OD of the microorganisms introduced into the plate in the absence of solutions. All of the tests were performed in triplicate.

\section{Results and Discussion}

Applying broth microdilution testing to a number of bacterial strains provides an estimate of the concentration that inhibits bacterial isolates and can indicate shifts in the susceptibility of bacterial populations to gold nanoparticles. This antimicrobial activity assay is therefore often the starting point for larger preclinical evaluations of novel antimicrobial agents.

To pursuit the broth microdilution testing, initially the gold nanoparticles were synthesized to obtain the right concentration, size and stability. Photodynamic effectivity was evaluated comparing the bacterial inhibition results obtained with and without light incubation.

\subsection{Synthesis of Curcumin gold nanoparticles (CurcAuNPs)}

The absorption spectrum of the solution measured after illumination by the $\mathrm{Hg}$ for 15 minutes is shown in Figure 1. As can be seen in this figure, the irradiated solution exhibits the plasmon resonance band typical for $\mathrm{Au}$ nanoparticles centered at $\sim 562 \mathrm{~nm}$.

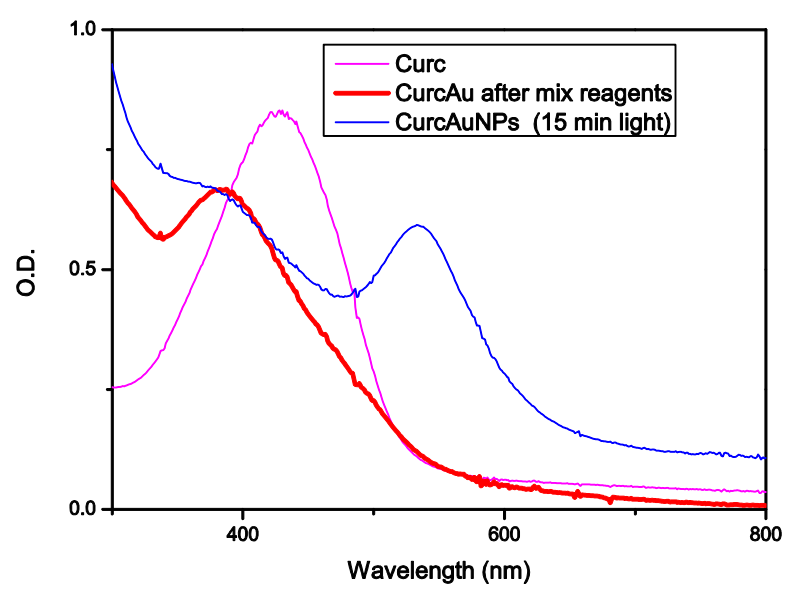

Figure 1. Absorption spectra of Curcumin and CurcAuNPs synthesised with $1.5 \mathrm{mg}$ of $\mathrm{HAuCl}_{4}, 3.2 \mathrm{mg}$ of Curcumin, PEG $(10 \mathrm{mg} / \mathrm{ml})$ in $100 \mathrm{~mL}$ milliQ water, and illumination for 15 $\min$.

TEM was used to characterize the size, shape and morphology of the synthesized CurcAuNPs. The TEM images of synthesized nanoparticles are shown in Figure 2. The morphology of CurcAuNPs is nearly spherical. The CurcAuNPs sizes ranged from 15 to $18 \mathrm{~nm}$, as observed in figure 2.

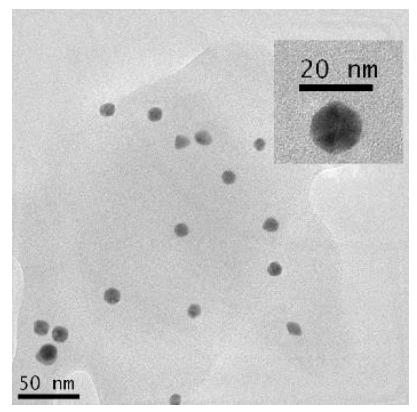

Figure 2. Gold nanostructures visualized by transmission electron microscopy of CurcAuNPs, with PEG and irradiated by 15 minutes of $\mathrm{Hg}$. 


\subsection{Antimicrobial activity}

A study on the sensitivity of bacteria to exposure to CurcAuNPs was performed. This study was achieved according to the microdilution test ${ }^{26}$ and provided results for the inhibition of growth of certain microorganisms.

The microdilution test provided quantitative results concerning the inhibition of bacterial growth, as obtained by reading the plates in a spectrophotometer. The data from the experiments performed in triplicate are shown in Figures 3 referring to the incubation periods of 18 hours, without and with PDT, respectively.

The results indicate that the gold nanoparticles strongly inhibited the development of Gram positive and Gram negative bacteria. After 18 hours of incubation with CurcAuNPs, inhibition of all of the tested strains where higher than incubation for concentrations higher that $15 \mu \mathrm{g} / \mathrm{mL}$. For $S$. aureus, the inhibition of which in the presence of the highest concentration of the antimicrobial agent was close to $98 \%$ at $30 \mu \mathrm{g} / \mathrm{mL}$. For S. epidermidis concentrations of 22.5 and $30 \mu \mathrm{g} / \mathrm{mL}$ of CurcAuNPs induces a change in absorption spectra of bacteria probably due to aptoptosis. The E. coli was the unique strain that showed good response for PDT and 30 $\mu \mathrm{g} / \mathrm{mL}$ of CurcAuNPs. Other light irradiation conditions must be studied to understand the PDT effect.

According to the literature, the interaction of gold nanoparticles is that the nanoparticles are capable of penetrating the inner bacterial cell can interact with DNA molecules through the phosphorus groups, leading to loss of the capacity for cell replication.

AuNPs mainly exert their antibacterial action in three ways ${ }^{27}: 1$ ) by changing membrane potential and inhibiting adenine triphosphate (ATP) synthase activities to lower the level of ATP, activating metabolic pathways that generate anabolic reactions. The ATPdependent mechanism essentially consists of pumping the antimicrobial ions that are present in the cytoplasm to the extracellular medium. 2) by inhibiting the subunit of ribosome for tRNA (transfer ribonucleic acid) binding, generating a blockade of the vital reactions in bacteria. 3) Some authors recently suggested that the inhibitory action occurs due to free radicals derived from the particle surface that are capable of damaging the cell membrane. The efflux pumps are recognized as one of the main forms of antibiotic resistance and metal ion mechanism. In the presence of a sensitizer, ROS (reactive oxygen species)-related process occurs summated to the other processes.

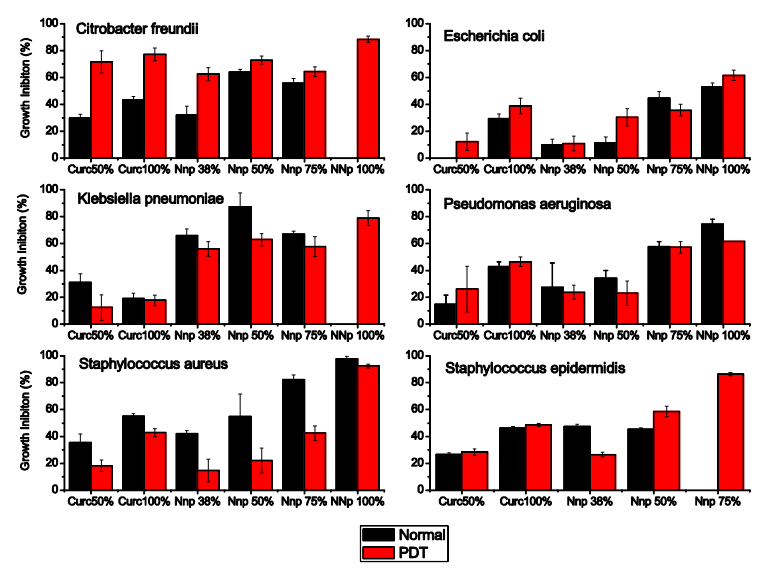

Figure 3. Growth inhibition versus CurcAuNps concentration graph: broth microdilution method, reading after 18 hours of incubation. In red with PDT and in blue without PDT.

\section{Conclusion}

In this paper, Curcumin gold nanoparticles were synthesized using a green chemistry method based on Curcumin, PEG and light. The preparation conditions were optimized using the results of absorption spectroscopy, and the antimicrobial effect of the resulting nanoparticles was tested.

The antimicrobial sensitivity test performed using the broth microdilution method provided quantitative data on the inhibitory effect of gold nanoparticles on microorganisms, which, in concentrations greater than $22.5 \mathrm{~g} / \mathrm{mL}$, revealed that the inhibition was close to $50 \%$ for period of 18 hours. Thus, the gold nanoparticles synthesized based on the concepts of the green chemistry methodology exhibited promising results regarding the development of the inhibitory action to different bacteria species. In general PDT, in the used illumination conditions, does not presented good improvements.

\section{Acknowledgements}

The National Council for Scientific and Technological Development (CNPq) is acknowledged for their financial support and Fapesp (grant 2014/069609), and Flávia Rodrigues de Oliveira Silva is acknowledged for providing the electron microscope support. Daniella dos Santos Courrol is fellowship of PAP, Secretariat of Health fellowship. 


\section{References}

[1] Y. Wang, Z. Lu, H. Wu and F. Lv, International Journal of Food Microbiology, vol. 136, pp. 71-74, 2009.

[2] R. L. Thangapazham, S. Sharad and R. K. Maheshwari, Biofactors, vol. 39, pp. 141-149, 2013.

[3] C. Syng-ai, A. L. Kumari and A. Khar, Molecular Cancer Therapeutics, vol. 3, pp. 1101-1108, 2004.

[4] R. A. Sharma, A. J. Gescher and W. P. Steward, European Journal of Cancer, vol. 41, pp. 1955-1968, 2005.

[5] T. A. Nguyen and A. J. Friedman, Journal of Drugs in Dermatology, vol. 12, pp. 1131-1137, 2013.

[6] J. J. Johnson and H. Mukhtar, Cancer Letters, vol. 255, pp. 170-181, 2007.

[7] P. Hu, P. Huang and M. W. Chen, Archives of Oral Biology, vol. 58, pp. 1343-1348, 2013.

[8] S. C. Gupta, S. Patchva and B. B. Aggarwal, Aaps Journal, vol. 15, pp. 195-218, 2013.

[9] R. K. Basniwal, H. S. Buttar, V. K. Jain and N. Jain, Journal of Agricultural and Food Chemistry, vol. 59, pp. 2056-2061, 2011.

[10] D. Rai, J. K. Singh, N. Roy and D. Panda, Biochemical Journal, vol. 410, pp. 147-155, 2008.

[11] B. S. Park, J. G. Kim, M. R. Kim, S. E. Lee, G. R. Takeoka, K. B. Oh and J. H. Kim, Journal of Agricultural and Food Chemistry, vol. 53, pp. 90059009, 2005.

[12] T. Rudrappa and H. P. Bais, Journal of Agricultural and Food Chemistry, vol. 56, pp. 1955-1962, 2008.

[13] A. Callegari, D. Tonti and M. Chergui, Nano Letters, vol. 3, pp. 1565-1568, 2003.

[14] S. Horikoshi, H. Abe, K. Torigoe, M. Abe and N. Serpone, Nanoscale, vol. 2, pp. 1441-1447, 2010.

[15] R. Gottesman, S. Shukla, N. Perkas, L. A. Solovyov, Y. Nitzan and A. Gedanken, Langmuir, vol. 27, pp. 720-726, 2011.

[16] H. T. Tung, I. G. Chen, I. M. Kempson, J. M. Song, Y. F. Liu, P. W. Chen, W. S. Hwang and Y. Hwu, ACS Appl Mater Interfaces, vol. 4, pp. 5930-5935, 2012.

[17] M. Roni, K. Murugan, C. Panneerselvam, J. Subramaniam, M. Nicoletti, P. Madhiyazhagan, D. Dinesh, U. Suresh, H. F. Khater, H. Wei, A. Canale, A. A. Alarfaj, M. A. Munusamy, A. Higuchi and G. Benelli, Ecotoxicol Environ Saf, vol. 121, pp. 31-38, 2015.

[18] N. Li, P. Zhao and D. Astruc, Angewandte ChemieInternational Edition, vol. 53, pp. 1756-1789, 2014.

[19] Y. Zhou, C. Y. Wang, Y. R. Zhu and Z. Y. Chen, Chemistry of Materials, vol. 11, pp. 2310-+, 1999.
[20] S. Dong, C. Tang, H. Zhou and H. Z. Zhao, Gold Bulletin, vol. 37, pp. 187-195, 2004.

[21] R. J. Tomita, R. A. de Matos, M. A. Vallim and L. C. Courrol, Journal of Photochemistry and Photobiology B-Biology, vol. 140, pp. 157-162, 2014.

[22] X. Mao, J. Kwon, H. Zhou and J. Lee, Current Applied Physics, vol. 15, pp. 1100-1105, 2015.

[23] R. K. Gangwar, V. A. Dhumale, D. Kumari, U. T. Nakate, S. W. Gosavi, R. B. Sharma, S. N. Kale and S. Datar, Materials Science \& Engineering C-Materials for Biological Applications, vol. 32, pp. 2659-2663, 2012.

[24] C. Sreelakshmi, N. Goel, K. K. R. Datta, A. Addlagatta, R. Ummanni and B. V. S. Reddy, Nanoscience and Nanotechnology Letters, vol. 5, pp. 1258-1265, 2013.

[25] I. Shlar, E. Poverenov, Y. Vinokur, B. Horev, S. Droby and V. Rodov, Nano-Micro Letters, vol. 7, pp. 68-79, 2015.

[26] P. S. f. A. S. Testing and T.-F. I. Supplement, NCCLS document M100-S25, Wayne, PA: National Committee of Clinical Laboratory Standards, 2012.

[27] Y. Cui, Y. Zhao, Y. Tian, W. Zhang, X. Lu and X. Jiang, Biomaterials, vol. 33, pp. 2327-2333, 2012. 